\title{
Surgical Outcomes of Minimally Invasive Stabilization for Spinal Fractures in Patients with Ankylosing Spinal Disorders
}

\author{
Kazuhiro Kai, Ko Ikuta, Keigo Masuda, Takahiro Kitamura, Hideyuki Senba, Satoshi Shidahara \\ Department of Orthopedic Surgery, Japanese Red Cross Society Karatsu Red Cross Hospital, Karatsu, Japan
}

\begin{abstract}
Study Design: A retrospective study.
Purpose: To evaluate the clinical and radiological outcomes of ankylosing spinal disorder (ASD) patients with spinal fractures treated by minimally invasive stabilization (MISt) using percutaneous pedicle screws (PPSs).

Overview of Literature: ASDs, such as ankylosing spondylitis (AS) and diffuse idiopathic skeletal hyperostosis (DISH), increase susceptibility to spinal fractures because of extremely decreased spinal flexibility. Such fractures tend to be unstable and, consequently, should be treated with multiple-segmental internal fixation. However, conventional internal fixation procedures can severely damage the soft tissue, resulting in severe hemorrhage. Therefore, MISt is the preferred approach to treat spinal fractures in ASD patients. Methods: Nine ASD patients (four males and five females; three AS and six DISH patients) with spinal fractures who were treated by MISt using PPSs, were reviewed from April 2009 to August 2016. One patient died of aspiration pneumonia during follow-up (FU), and the remaining eight patients underwent clinical and radiological evaluation.

Results: The mean age at surgery was 79.6 years (range, 68-95 years). The mean duration of postoperative FU was 14.2 months (range, 3-30 months). All treated fractures were anterior and posterior element injuries with distraction. Three patients presented delayed onset preoperative neurological deficit following trauma. The mean operation time was 179.6 minutes (range, 92-340 minutes). The mean hemorrhage was $103.6 \mathrm{~mL}$ (range, unquantifiable to $480 \mathrm{~mL}$ ). Radiological evaluations at FU showed preservation of the acceptable postoperative correction of the fractured vertebra, as there were no re-collapses of the fractured vertebrae during FU.

Conclusions: ASD patients must be acknowledged as highly susceptible to unstable spinal fractures, even after relatively mild trauma. MISt using PPSs may be an effective treatment for spinal fractures in such patients.
\end{abstract}

Keywords: Spondylarthritis; Ankylosing spondylitis; Diffuse idiopathic skeletal hyperostosis; Minimally invasive surgical procedures; Pedicle screws

\section{Introduction}

Ankylosing spinal disorders (ASDs), including ankylosing spondylitis (AS) and diffuse idiopathic skeletal hyperostosis (DISH), remarkably increase susceptibility to unstable spinal fractures, even following relatively mild trauma, because of an extreme reduction of spinal flexibility [1-5]. Such fractures often cannot be identified on plain radiographs, which lead to a delayed diagnosis. Furthermore, these fractures are often accompanied by delayed neuro-

Received Aug 9, 2017; Revised Sep 30, 2017; Accepted Oct 3, 2017

Corresponding author: Kazuhiro Kai

Department of Orthopedic Surgery, Japanese Red Cross Society Karatsu Red Cross Hospital, 2430 Watada, Karatsu City, Saga 847-8588, Japan

Tel: +81-0952-72-5111, Fax:+81-0955-73-9530,E-mail: hiro_stylish.0305@me.com 
logical deficits [6-9].

The ideal surgical treatment for these unstable fractures remains controversial. Although multiple-segmental internal fixation is usually recommended [7,9-11], conventional internal fixation procedures can severely damage the soft tissue, resulting in severe hemorrhage [12]. Recently, minimally invasive stabilization (MISt) has been used to treat several disorders, including degenerative diseases, trauma, tumors, and infection.

The aim of this study was to evaluate the clinical and radiological outcomes of ASD patients with spinal fractures treated by MISt using percutaneous pedicle screws (PPSs).

\section{Materials and Methods}

Between April 2009 and August 2016, we retrospectively reviewed patients with spinal fractures who were diagnosed with either AS or DISH at Karatsu Red Cross Hospital. Twelve patients underwent surgical treatment, among whom three were excluded as they were treated with conventional internal fixation procedures. Therefore, nine patients (three AS and six DISH patients) who were treated by MISt using PPSs were included in the study. AS and DISH diagnosis was according to clinical and radiological findings [4,5]. Data were obtained from medical records and operation notes. Assessment of postoperative health-related quality-of-life was according to the EuroQol-5D (EQ-5D) [13], which was administered as a medical interview, conducted at the final follow-up (FU).

All patients underwent preoperative plain radiography and computed tomography (CT). Postoperative plain radiographs were obtained in the prone position immediately after surgery and in a standing position at the final FU. The sagittal Cobb's angle and the fractured vertebra's anterior and posterior heights were measured before and after surgery and at the final FU. The sagittal Cobb's angle was defined as the angle formed between a line drawn parallel to the superior endplate of the vertebra above the fracture and a line drawn parallel to the inferior endplate of vertebra below the fracture (Fig. 1). Kyphotic angulation was described with positive values. The anterior and posterior vertebral heights were measured as the distance between the upper and lower endplates at the anterior and posterior walls of the fractured vertebral body, respectively. One patient died of aspiration pneumonia 3 months after surgery, and the remaining eight patients underwent clinical and radiological evaluation.

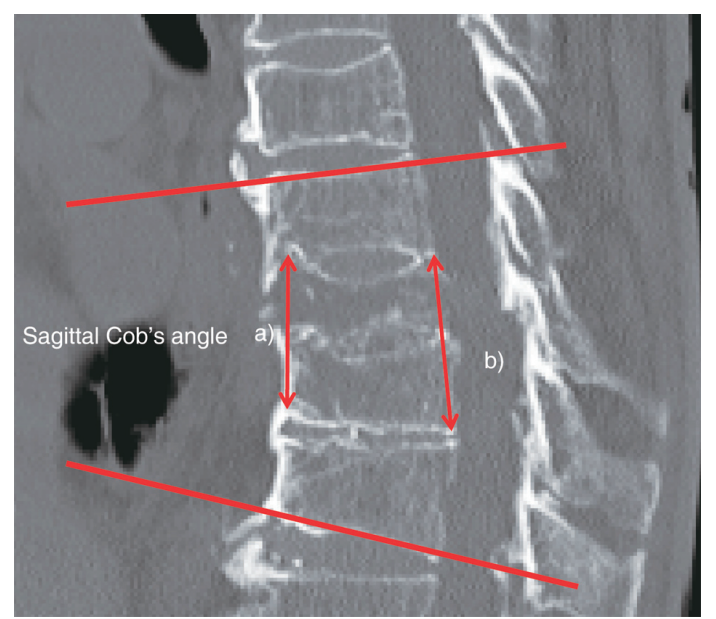

Fig. 1. Sagittal Cobb's angle. ${ }^{\text {al }}$ Anterior vertebral height. ${ }^{\text {blPosterior }}$ vertebral height.

\section{Surgical technique}

MISt was performed by spinal surgeons with the patients in the prone position on a radiolucent table under general anesthesia. Intraoperative anteroposterior and lateral images were obtained using fluoroscopy. Skin incisions were marked laterally over each chosen pedicle using fluoroscopy. A Jamshidi needle was inserted into the vertebral body through the pedicle, and a guide wire was inserted through the needle into the vertebral body. The needle was carefully removed, and cannulated cancellous screws were inserted over the guide wires. Percutaneous vertebroplasty using hydroxyapatite was performed in the posterior instrumentation to achieve anterior column support. This was not performed in cases where the structural stability of the anterior column was evident.

According to the AO-Magerl classification system, fracture types were classified as either $\mathrm{AO}$ type $\mathrm{B} 1$ or $\mathrm{AO}$ type $\mathrm{B} 3$. AO type $\mathrm{B} 1$ involved anterior vertebral body collapse and required vertebroplasty to prevent additional vertebral body collapse. AO type B3 involved disruption of the anterior column, and the gap was filled by percutaneous vertebroplasty using hydroxyapatite. The used rods conformed to the shape of the patient's spinal column and were placed over the screws. No spinal alignment corrections, such as compression or distraction, were performed, as there was no obvious malalignment.

\section{Results}

Four patients were male and five were female, and the 
mean age at surgery was $79.5 \pm 9.5$ years (range, 68-95 years). The mean FU duration was 14.2 \pm 9.7 months (range, 3-30 months). The injury mechanisms included two cases of traffic injuries, three cases of falls from a height and four cases of ground-level falls. A total of 10 spinal fractures were detected, as one patient presented two fractures (case 5).

All fractures were anterior and posterior element injuries with distraction (AO type B1:2, AO type B3: 7). Preoperative back pain was reported by all patients, whereas three patients presented preoperative neurological deficits with delayed onset following trauma. Six patients had been transferred from another hospital and had a delayed diagnosis. The mean interval between the injury and the operation was $32.6 \pm 47.2$ days (range, $5-150$ days).

Patient demographics and clinical information are presented in Table 1 . The mean surgery duration was $179.5 \pm 78.8$ minutes (range, $92-340$ minutes) and the mean hemorrhage was $103.6 \pm 152.9 \mathrm{~mL}$ (range, unquantifiable to $480 \mathrm{~mL}$ ). One patient had an infection of the surgical site (case 7). The mean length of hospital stay was $76.3 \pm 47.0$ days (range, 37-178 days). Eight patients had bony union, excluding one patient who died of aspiration pneumonia during FU. Three patients complained of mild back pain postoperatively. The mean EQ-5D utility score was $0.70 \pm 0.29$ (range, $0.132-1$ ). The surgical treatment and outcomes are shown in Table 2.

Radiological assessments revealed that the mean difference between the postoperative and final FU sagittal Cobb angle was $2^{\circ}$. FU evaluation indicated that the surgical correction of the fractured vertebra was acceptable, with no cases of obvious re-collapse of the fractured vertebra (Table 3). In one patient (case 7), the hardware was removed 3 months after surgery due to skin problems.

Unfortunately, no patients were treated for osteoporosis. All patients wore a soft corset and one patient (case 3) temporarily wore a hard corset.

\section{Case 3}

A 72-year-old man with AS had back pain after a groundlevel fall. He visited a clinic and was diagnosed with a vertebral compression fracture. Five months after injury the back pain worsened, and he visited our hospital. A lateral plain radiograph showed an $\mathrm{L} 4$ unstable fracture (Fig. $2 \mathrm{~A}$ ), and upon CT and magnetic resonance imaging (MRI) the patient was diagnosed with an undisplaced 3-column

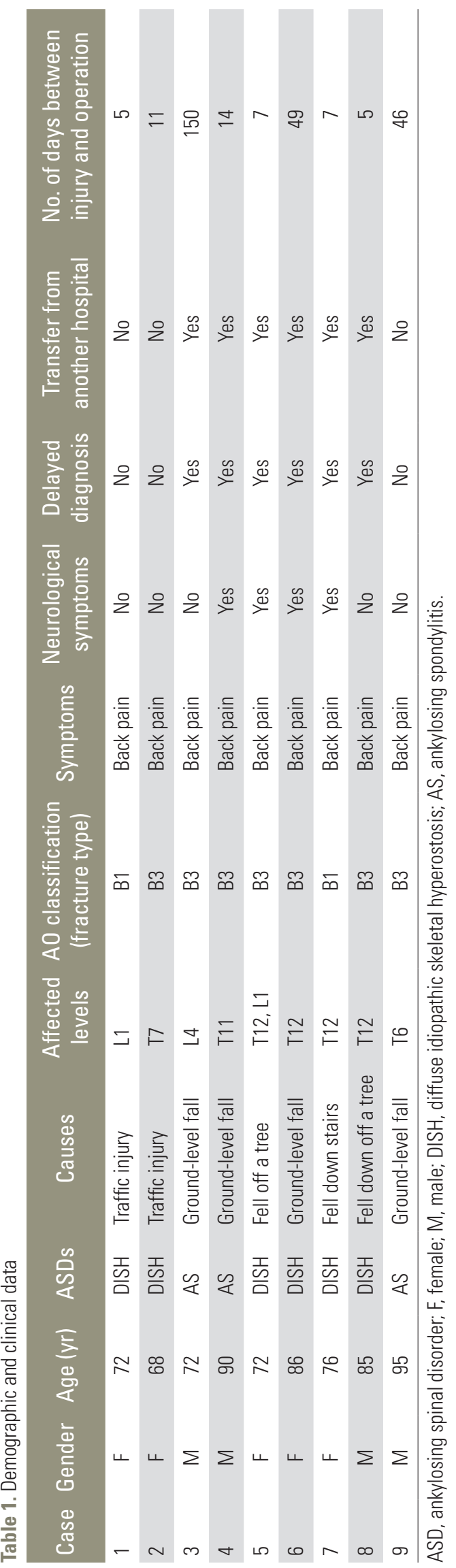




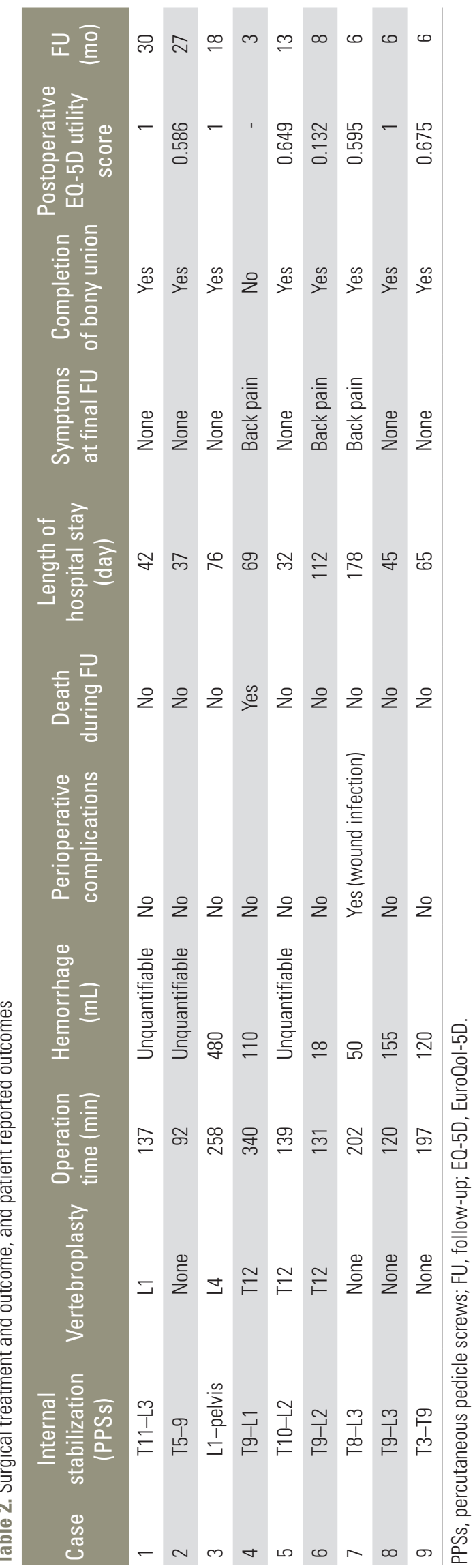

fracture at the L4 (Fig. 2B, C). Corrective surgery was performed 150 days after the initial injury (Fig. 2D, E). The procedure was conducted with the patient in the prone position under general anesthesia. Percutaneous posterior stabilization was performed between L2 and S1, and percutaneous vertebroplasty using hydroxyapatite blocks was performed at the fractured L4. A mini-open midline skin incision exposing the dorsal foramina of the sacrum was open, through which two sacral alar-iliac (SAI) screws were inserted. Rods were inserted percutaneously and connected to the S1 pedicle screws and the SAI screws via the mini-open midline skin incision, because those screws are close together and thus were difficult to connect. Following surgery, the patient wore a hard corset for 6 weeks, after which he wore a thoracolumbar soft corset. Completed bony union was confirmed 4 months after surgery (Fig. 2F). At the 18-month FU, the back pain had improved, and the patient was asymptomatic.

\section{Discussion}

\section{Delayed diagnosis}

Several studies of ASD-related fractures report a delayed diagnosis. Caron et al. [6] report that this is the case for $19 \%$ ASD-related fractures, and that $81 \%$ patients with a delayed diagnosis present neurological deficits. In the present study, a delayed diagnosis was found for $75 \%$ patients (six of nine), and $50 \%$ of those (three of six) presented neurological deficits. In ASD patients, spinal fractures are often missed as they are typically caused by low-energy injuries, with negative plain radiograph findings. An et al. [14] showed that in ASD cases, plain radiographs of the spine may fail to reveal a fracture due to the distorted anatomy, ossified ligaments, and artifacts; thus, an initial CT or MRI of the whole spine is recommended. Furthermore, a delayed diagnosis may arise from a lack of knowledge regarding ASD-related fractures. Indeed, previous studies have reported that ASD patients are often misdiagnosed as having vertebral compression fractures. Therefore, ASD patients should be acknowledged as being at high risk for spinal fractures, to avoid delayed diagnosis.

\section{Minimally invasive stabilization}

ASD greatly increases susceptibility to unstable spinal 
Table 3. Change of radiographic parameters

\begin{tabular}{lccc} 
Variable & Preoperative & Postoperative & Final follow-up \\
Sagittal Cobb angle $\left(^{\circ}\right)$ & $9.3 \pm 12.3$ & $9.3 \pm 13.3$ & $11.5 \pm 17.0$ \\
Anterior vertebral height $(\mathrm{mm})$ & $24.0 \pm 3.58$ & $28.6 \pm 6.29$ & $22.5 \pm 7.44$ \\
\hline Posterior vertebral height $(\mathrm{mm})$ & $27.0 \pm 3.80$ & $28.7 \pm 5.38$ & $28.0 \pm 5.83$ \\
\hline
\end{tabular}

Values are presented as mean \pm standard deviation.
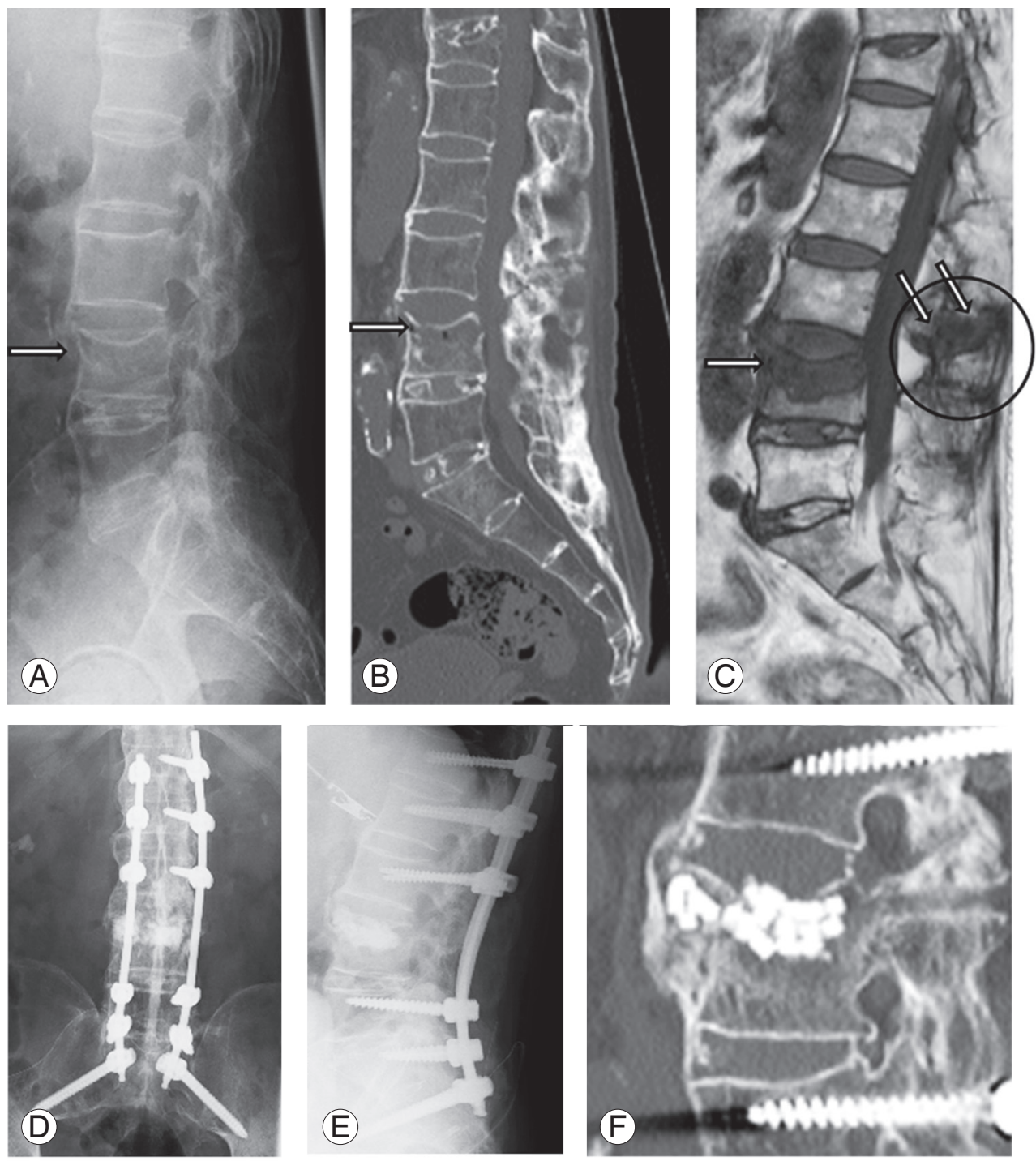

Fig. 2. (A) Preoperative lateral plain radiograph showed instability at $L 4$ (hyperextension injury, arrow). (B, C) Preoperative CT and magnetic resonance imaging showing an undisplaced 3-column fracture at L4 (arrow). (D, E) Postoperative plain radiographs. (F) Postoperative CT sagittal image with L4 showing a bony union at 4 months after surgery. CT, computed tomography.

fractures, due to an extreme reduction of spinal flexibility. The ideal surgical treatment for such unstable fractures remains controversial. Several studies have reported that multiple-segmental internal fixation is recommended to surgically correct ASD-related fractures [6,7,10,11] although such techniques are very time-consuming and invasive. Indeed, Westerveld et al. [12] described a mean open surgery duration of 264 minutes for AS patients and 
203 minutes for DISH patients, with a mean hemorrhage of 960 and $788 \mathrm{~mL}$, respectively. In contrast, MISt using PPSs involves lower hemorrhage and damage to soft tissue and requires a shorter operative time. Yeoh et al. [11] found that MISt for AS was generally associated with low levels of hemorrhage, which decreased the requirement for blood transfusions. Our mean operative time was shorter, and our mean hemorrhage was considerably lower than the values reported by Westerveld et al. [12], indicating that minimally invasive surgery may be an effective treatment for ASD-related spinal fractures. In fact, all cases included in the present study were successfully treated with MISt using PPSs.

MISt using PPSs is usually a temporary fixation, and hardware removal is recommended for patients that achieve complete bone union. However, bone grafting and removal of the hardware are often not necessary, because the spine of ASD patients usually presents multi-level vertebral bony fusions. At our institution hardware removal is performed at the patients' discretion, provided that a complete bone union is achieved. Furthermore, although we were unable to directly view the site at which the pedicle screws had been inserted, our findings indicate that MISt using PPSs is a safe surgical procedure if a surgeon receives sufficiently training before surgery. Posterior stabilization using PPSs in the thoracic and lumbar spine has become increasingly popular because of its safety and achieved clinical results [15]. Ikeuchi and Ikuta [16] evaluated the accuracy of PPSs placement as compared with that of conventional open techniques. They reported no statistically significant differences regarding the accuracy of the two methods, and indicated that PPSs could be ideally inserted without complications [16]. Although MISt using PPSs is the most suitable surgical procedure for

Table 4. Advantages and disadvantages of MISt and open surgery

\begin{tabular}{lll} 
& \multicolumn{1}{c}{ Advantages } & \multicolumn{1}{c}{ Disadvantages } \\
Open & Easy for bone grafting & $\begin{array}{l}\text { Long operative time } \\
\text { Damage to soft tissue } \\
\text { Hemorrhage } \\
\text { Wound pain }\end{array}$ \\
& & $\begin{array}{l}\text { Exposure to radiation } \\
\text { MISt } \quad \begin{array}{l}\text { Shorten operative time } \\
\text { Decreased damage to soft tissue }\end{array}\end{array}$ \\
& $\begin{array}{l}\text { A small amount of hemorrhage } \\
\text { Mild wound pain }\end{array}$ & \\
\hline
\end{tabular}

MISt, minimally invasive stabilization. treating spinal fracture in ASD patients, it is not without disadvantages. Table 4 summarizes the advantages and disadvantages of MISt and open surgery.

One disadvantage of MISt is radiation exposure. Using fluoroscopy is vital to ensure a safe and accurate insertion of the PPSs, as it depends on visualizing intraoperative radiographic images. However, Yeoh et al. [11] reported that obtaining good-quality intraoperative images was difficult, especially for the upper thoracic spine, and this difficulty is increased in the spine of ASD patients. Radiation exposure is harmful to surgeons, nurses, and patients. Surgeons should therefore train sufficiently before surgery, and spend more time with preoperative preparations, to ensure that the radiation exposure duration is minimized.

\section{Vertebroplasty}

Several surgical techniques to treat ASD-related spinal fractures have used percutaneous posterior instrumentation alone $[10,14,17,18]$. However, few reports have described percutaneous vertebroplasty using hydroxyapatite blocks [12]. We combined percutaneous vertebroplasty using hydroxyapatite with posterior instrumentation, to achieve anterior column support. Toyone et al. [19] stated that hydroxyapatite grafting to the fractured body allowed to support the anterior column, thus preventing kyphosis in treating vertebral body compressions. However, most patients in our study had hyperextension injuries. In such cases, the disruption of the anterior column is worsened when the patient is in the prone position, on a radiolucent operating table. In our study, this was compensated for by performing percutaneous vertebroplasty using hydroxyapatite. Consequently, surgical correction of the fractured vertebra was acceptable at the final FU with no cases of obvious re-collapse of the fractured vertebra (Table 3). No instrumentation failure was observed, and we acknowledge that these results were achieved because of an adequate support of the anterior column. Therefore, percutaneous vertebroplasty using hydroxyapatite and percutaneous posterior instrumentation may be a useful surgical procedure.

\section{Reduction of spinal alignment}

To minimize implant-loosening risk, it is important to improve the fracture reduction and sagittal alignment. However, improving reduction of hyperextension spine 
injuries in ASD patients may be difficult, as the disruption of the anterior column is worsened when the patients are in the prone position. Lindtner et al. [20] described a new treatment concept for the surgical management of hyperextension injuries of the thoracolumbar spine in patients with ASD, involving percutaneous less rigid posterior instrumentation. Fracture reduction by postoperative mobilization is feasible, and seems to facilitate adequate reduction and restoration of the preinjury sagittal alignment [20]. In our study, there were no cases of obvious malalignment, although the disruption of the anterior column did worsen in some cases. Usually, in the absence of obvious malalignment, percutaneous posterior fixation without reduction is performed. Oppositely, in the presence of obvious malalignment improving fracture reduction and sagittal alignment may be necessary.

\section{Conclusions}

ASD patients must be acknowledged as highly susceptible to unstable spinal fractures, even after relatively mild trauma. MISt using PPSs may be an effective alternative to multiple-segmental internal fixation for the treatment of ASD-related unstable spinal fractures.

\section{Conflict of Interest}

No potential conflict of interest relevant to this article was reported.

\section{Acknowledgments}

We acknowledge the past and present work members of Japanese Red Cross Society Karatsu Red Cross Hospital.

\section{References}

1. Burkus JK, Denis F. Hyperextension injuries of the thoracic spine in diffuse idiopathic skeletal hyperostosis: report of four cases. J Bone Joint Surg Am 1994;76:237-43.

2. Forestier J, Rotes-Querol J. Senile ankylosing hyperostosis of the spine. Ann Rheum Dis 1950;9:321-30.

3. Paley D, Schwartz M, Cooper P, Harris WR, Levine AM. Fractures of the spine in diffuse idiopathic skeletal hyperostosis. Clin Orthop Relat Res $1991 ;(267): 22-32$.
4. Resnick D, Shaul SR, Robins JM. Diffuse idiopathic skeletal hyperostosis (DISH): Forestier's disease with extraspinal manifestations. Radiology 1975;115:51324.

5. Weinstein PR, Karpman RR, Gall EP, Pitt M. Spinal cord injury, spinal fracture, and spinal stenosis in ankylosing spondylitis. J Neurosurg 1982;57:609-16.

6. Caron T, Bransford R, Nguyen Q, Agel J, Chapman J, Bellabarba C. Spine fractures in patients with ankylosing spinal disorders. Spine (Phila Pa 1976) 2010;35:E458-64.

7. Hsieh PC, Koski TR, Sciubba DM, et al. Maximizing the potential of minimally invasive spine surgery in complex spinal disorders. Neurosurg Focus 2008;25:E19.

8. Schiefer TK, Milligan BD, Bracken CD, et al. In-hospital neurologic deterioration following fractures of the ankylosed spine: a single-institution experience. World Neurosurg 2015;83:775-83.

9. Westerveld LA, Verlaan JJ, Oner FC. Spinal fractures in patients with ankylosing spinal disorders: a systematic review of the literature on treatment, neurological status and complications. Eur Spine J 2009;18:145-56.

10. Kruger A, Frink M, Oberkircher L, El-Zayat BF, Ruchholtz S, Lechler P. Percutaneous dorsal instrumentation for thoracolumbar extension-distraction fractures in patients with ankylosing spinal disorders: a case series. Spine J 2014;14:2897-904.

11. Yeoh D, Moffatt T, Karmani S. Good outcomes of percutaneous fixation of spinal fractures in ankylosing spinal disorders. Injury 2014;45:1534-8.

12. Westerveld LA, van Bemmel JC, Dhert WJ, Oner FC, Verlaan JJ. Clinical outcome after traumatic spinal fractures in patients with ankylosing spinal disorders compared with control patients. Spine J 2014;14:729-40.

13. EuroQol Group. EuroQol: a new facility for the measurement of health-related quality of life. Health Policy 1990;16:199-208.

14. An SB, Kim KN, Chin DK, Kim KS, Cho YE, Kuh SU. Surgical outcomes after traumatic vertebral fractures in patients with ankylosing spondylitis. J Korean Neurosurg Soc 2014;56:108-13.

15. Heintel TM, Berglehner A, Meffert R. Accuracy of percutaneous pedicle screws for thoracic and lumbar spine fractures: a prospective trial. Eur Spine J 


\section{3;22:495-502.}

16. Ikeuchi H, Ikuta K. Accuracy of pedicle screw insertion in the thoracic and lumbar spine: a comparative study between percutaneous screw insertion and conventional open technique. Arch Orthop Trauma Surg 2016;136:1195-202.

17. Nayak NR, Pisapia JM, Abdullah KG, Schuster JM. Minimally invasive surgery for traumatic fractures in ankylosing spinal diseases. Global Spine J 2015;5:266-73.

18. Sedney CL, Daffner SD, Obafemi-Afolabi A, et al. A comparison of open and percutaneous techniques in the operative fixation of spinal fractures associated with ankylosing spinal disorders. Int J Spine Surg 2016;10:23.
19. Toyone T, Tanaka T, Kato D, Kaneyama R, Otsuka M. The treatment of acute thoracolumbar burst fractures with transpedicular intracorporeal hydroxyapatite grafting following indirect reduction and pedicle screw fixation: a prospective study. Spine (Phila $\mathrm{Pa}$ 1976) 2006;31:E208-14.

20. Lindtner RA, Kammerlander C, Goetzen M, et al. Fracture reduction by postoperative mobilisation for the treatment of hyperextension injuries of the thoracolumbar spine in patients with ankylosing spinal disorders. Arch Orthop Trauma Surg 2017;137:53141. 